\title{
APPROXIMATION OF PASSAGE TIMES OF $\gamma$-REFLECTED PROCESSES WITH FBM INPUT
}

\author{
ENKELEJD HASHORVA* AND \\ LANPENG JI, ** University of Lausanne
}

\begin{abstract}
Define a $\gamma$-reflected process $W_{\gamma}(t)=Y_{H}(t)-\gamma \inf _{s \in[0, t]} Y_{H}(s), t \geq 0$, with input process $\left\{Y_{H}(t), t \geq 0\right\}$, which is a fractional Brownian motion with Hurst index $H \in$ $(0,1)$ and a negative linear trend. In risk theory $R_{\gamma}(u)=u-W_{\gamma}(t), t \geq 0$, is referred to as the risk process with tax payments of a loss-carry-forward type. For various risk processes, numerous results are known for the approximation of the first and last passage times to 0 (ruin times) when the initial reserve $u$ goes to $\infty$. In this paper we show that, for the $\gamma$-reflected process, the conditional (standardized) first and last passage times are jointly asymptotically Gaussian and completely dependent. An important contribution of this paper is that it links ruin problems with extremes of nonhomogeneous Gaussian random fields defined by $Y_{H}$, which we also investigate.
\end{abstract}

Keywords: Gaussian approximation; passage time; $\gamma$-reflected process; workload process; risk process with tax; fractional Brownian motion; Piterbarg constant; Pickands constant

2010 Mathematics Subject Classification: Primary 60G15

Secondary $60 \mathrm{G} 70$

\section{Introduction and main result}

Let $\left\{X_{H}(t), t \geq 0\right\}$ be a standard fractional Brownian motion (FBM) with Hurst index $H \in(0,1)$, meaning that $X_{H}$ is a centered Gaussian process with almost surely continuous sample paths and covariance function

$$
\operatorname{cov}\left(X_{H}(t), X_{H}(s)\right)=\frac{1}{2}\left(t^{2 H}+s^{2 H}-|t-s|^{2 H}\right), \quad t, s \geq 0 .
$$

We will define the $\gamma$-reflected process with input process $Y_{H}(t)=X_{H}(t)-c t$ by

$$
W_{\gamma}(t)=Y_{H}(t)-\gamma \inf _{s \in[0, t]} Y_{H}(s), \quad t \geq 0,
$$

where $\gamma \in[0,1]$ and $c>0$ are two fixed constants.

Motivations for studying $W_{\gamma}$ come from both risk and queueing theory. For instance, in queueing theory $W_{1}$ is the so-called workload process (or queue length process); see, e.g. Harrison (1985), Asmussen (1987), Zeevi and Glynn (2000), Whitt (2002), and Awad and Glynn (2009). In advanced risk theory the process $R_{\gamma}(t)=u-W_{\gamma}(t), t \geq 0, u \geq 0$, is referred to as the risk process with tax payments of a loss-carry-forward type; see, e.g. Asmussen and Albrecher (2010). Recently, Hashorva et al. (2013) studied the asymptotics of the probability

Received 26 June 2013; revision received 15 October 2013.

* Postal address: Department of Actuarial Science, University of Lausanne, UNIL-Dorigny 1015 Lausanne, Switzerland.

** Email address: jilanpeng@126.com 
$\mathbb{P}\left\{\sup _{t \in[0, T]} W_{\gamma}(t)>u\right\}$ as $u \rightarrow \infty$ for both $T<\infty$ and $T=\infty$. Continuing the study of the aforementioned paper in this contribution we will investigate the approximations of the first and last passage times of $W_{\gamma}$. Specifically, define the first and last passage times of $W_{\gamma}$ to a constant threshold $u>0$ by

$$
\tau_{1}(u)=\inf \left\{t \geq 0, W_{\gamma}(t)>u\right\} \quad \text { and } \quad \tau_{2}(u)=\sup \left\{t \geq 0, W_{\gamma}(t)>u\right\},
$$

respectively (here we use the convention that $\inf \{\varnothing\}=\infty$ and $\sup \{\varnothing\}=0$ ). Furthermore, define $\tau_{1}^{*}(u), \tau_{2}^{*}(u), u>0$, in the same probability space such that

$$
\left(\tau_{1}^{*}(u), \tau_{2}^{*}(u)\right) \stackrel{\mathrm{D}}{=}\left(\tau_{1}(u), \tau_{2}(u)\right) \mid\left(\tau_{1}(u)<\infty\right),
$$

where $\stackrel{\text { D }}{=}$ denotes equality in distribution.

The first and last passage times of Gaussian processes conditioned on the event $\left\{\tau_{1}(u)<\infty\right\}$ are analysed in Hüsler and Piterbarg (2008) and Hüsler and Zhang (2008) when $\gamma=0$. Therein, the Guassian approximations of both $\tau_{1}^{*}(u)$ and $\tau_{2}^{*}(u)$ are derived. First passage times (sometimes called ruin times) are also studied extensively in the framework of insurance risk processes; see the recent articles Griffin and Maller (2012), Griffin (2013), Griffin et al. (2013), and Dȩbicki et al. (2014), and the monographs Embrechts et al. (1997) and Asmussen and Albrecher (2010) for approximations of ruin times of various risk processes. In our framework, $\tau_{1}^{*}(u)$ can be interpreted as the conditional ruin time of the FBM risk process with tax payments of a loss-carry-forward type. With motivation from the aforementioned contributions, this paper is concerned with the Gaussian approximation of the random vector $\left(\tau_{1}^{*}(u), \tau_{2}^{*}(u)\right)$. For the derivation of the tail asymptotics of $\sup _{t \in[0, T]} W_{\gamma}(t)$, Hashorva et al. (2013) showed that the investigation of the supremum of certain nonstationary Gaussian random fields is crucial. One key merit of our problem of approximating the joint distribution function of $\left(\tau_{1}^{*}(u), \tau_{2}^{*}(u)\right)$ is that it leads, as in the case of the analysis of the tail asymptotics of $\sup _{t \in[0, T]} W_{\gamma}(t)$, to an interesting unsolved problem of asymptotic theory of Gaussian random fields. Although the latter investigation was not initially in the scope of this paper, the corresponding result derived in Theorem 2.1 is important for various theoretical questions. Next, set

$$
A(u)=\frac{H^{H+1 / 2}}{(1-H)^{H+1 / 2} c^{H+1}} u^{H} \quad \text { and } \quad \tilde{t}_{0}=\frac{H}{c(1-H)}
$$

and denote by $\stackrel{\mathrm{D}}{\rightarrow}$ ' and $\stackrel{\stackrel{\mathbb{P}}{\rightarrow}}{ }$ ' the convergences in distribution and in probability, respectively. Furthermore, let $\mathcal{N}$ be an $N(0,1)$ random variable. Our principal result is the following theorem.

Theorem 1.1. Let the $\gamma$-reflected process $\left\{W_{\gamma}(t), t \geq 0\right\}$ be given as in (1.1) with $\gamma \in(0,1)$, and let $\tau_{1}^{*}(u)$ and $\tau_{2}^{*}(u)$ be defined as in (1.2). Then, as $u \rightarrow \infty$,

$$
\left(\frac{\tau_{1}^{*}(u)-\tilde{t}_{0} u}{A(u)}, \frac{\tau_{2}^{*}(u)-\tilde{t}_{0} u}{A(u)}\right) \stackrel{\mathrm{D}}{\rightarrow}(\mathcal{N}, \mathcal{N}) .
$$

Remarks. (a) The joint convergence in (1.3) implies that $\left(\tau_{2}^{*}(u)-\tau_{1}^{*}(u)\right) / A(u) \stackrel{\mathbb{P}}{\rightarrow} 0$ as $u \rightarrow \infty$.

(b) For any $u \geq 0, \mathbb{P}\left\{\tau_{1}(u)<\infty\right\}=1$ when $\gamma=1$ (cf. Duncan and Jin (2008)), which is the reason for considering only the case that $\gamma \in(0,1)$. Under the latter assumption on $\gamma$, we further 
have $\mathbb{P}\left\{\tau_{2}(u)<\infty \mid \tau_{1}(u)<\infty\right\}=1$, which follows from the fact that $\lim _{t \rightarrow \infty} W_{\gamma}(t)=-\infty$ almost surely since in view of Remark 5 of Kozachenko et al. (2014)

$$
\lim _{t \rightarrow \infty} \frac{\sup _{s \in[0, t]}\left|X_{H}(s)\right|}{t}=0 \quad \text { for all } H \in(0,1) .
$$

(c) It is somewhat surprising that the Gaussian approximation of the conditional first and last passage times does not involve the reflection constant $\gamma$.

The rest of the paper is organized as follows. In the next section we present a key result on the supremum of some Gaussian random fields defined by $Y_{H}$ and then provide the proof of Theorem 1.1. Section 3 is dedicated to the proof of Theorem 2.1. A variant of the Piterbarg lemma suitable for Gaussian random fields is presented in Appendix A.

\section{Further results and the proof of Theorem 1.1}

Following the idea of Hüsler and Piterbarg (1999), (2008), and as discussed in Hashorva et al. (2013), it is convenient to introduce the following family of Gaussian random fields:

$$
Y_{u}(s, t):=\frac{X_{H}(u t)-\gamma X_{H}(u s)}{(1+c t-c \gamma s) u^{H}}, \quad s, t \geq 0, u>0 .
$$

The variance function of $\left\{Y_{u}(s, t), s, t \geq 0\right\}$ is given by

$$
V_{Y}^{2}(s, t)=\frac{(1-\gamma) t^{2 H}+\left(\gamma^{2}-\gamma\right) s^{2 H}+\gamma(t-s)^{2 H}}{(1+c t-c \gamma s)^{2}}, \quad s, t \geq 0 .
$$

Moreover, on the set $\{(s, t): 0 \leq s \leq t<\infty\}$, it attains its maximum at the unique point $\left(0, \tilde{t}_{0}\right)$ with $\tilde{t}_{0}=H / c(1-H)$, and, furthermore,

$$
V_{Y}\left(0, \tilde{t}_{0}\right)=\frac{H^{H}(1-H)^{1-H}}{c^{H}} .
$$

By the change of variables $t=t^{\prime} u$ and $s=s^{\prime} u$, and noting that the distribution of $Y_{u}$ does not depend on $u$, we obtain

$$
\begin{aligned}
\mathbb{P}\left\{\tau_{1}(u)<\infty\right\} & =\mathbb{P}\left\{\text { there exists } t \in[0, \infty) \text { such that } W_{\gamma}(t)>u\right\} \\
& =\mathbb{P}\left\{\text { there exists } t^{\prime} \in[0, \infty) \text { such that } Y_{u}\left(s^{\prime}, t^{\prime}\right)>u^{1-H} \text { for some } s^{\prime} \in\left[0, t^{\prime}\right]\right\} \\
& =\mathbb{P}\left\{\text { there exists } t \in[0, \infty) \text { such that } Y(s, t)>u^{1-H} \text { for some } s \in[0, t]\right\},
\end{aligned}
$$

where

$$
Y(s, t):=\frac{X_{H}(t)-\gamma X_{H}(s)}{1+c(t-\gamma s)}, \quad s, t \geq 0 .
$$

In order to complete the proof of Theorem 1.1, we need to know the tail asymptotic behavior of the supremum of the Gaussian random field $Y$ over a region which might depend on $u$. Therefore, we will first investigate the tail asymptotic behavior of the supremum of certain nonstationary Gaussian random fields (including $Y$ as a special case) over a region depending on $u$ in Theorem 2.1 followed then by the proof of Theorem 1.1. 
Hereafter, we assume that all considered Gaussian random fields (or processes) have almost surely continuous sample paths. We need to introduce some more notation, starting with the well-known Pickands constant $\mathscr{H}_{\alpha}$ given by

$$
\mathscr{H}_{\alpha}:=\lim _{T \rightarrow \infty} \frac{1}{T} \mathscr{H}_{\alpha}[0, T] \in(0, \infty), \quad \alpha \in(0,2],
$$

where

$$
\mathscr{H}_{\alpha}[0, T]=\mathbb{E}\left(\exp \left(\sup _{t \in[0, T]}\left(\sqrt{2} B_{\alpha}(t)-t^{\alpha}\right)\right)\right), \quad T \in(0, \infty),
$$

with $\left\{B_{\alpha}(t), t \geq 0\right\}$ an FBM with Hurst index $\alpha / 2 \in(0,1]$. It is known that $\mathscr{H}_{1}=1$ and $\mathscr{H}_{2}=1 / \sqrt{\pi}$; see Pickands (1969), Albin (1990), Piterbarg (1996), Dębicki (2002), Dębicki et al. (2003), Mandjes (2007), Dȩbicki and Mandjes (2011), and Dieker and Yakir (2014) for various properties of Pickands' constant and its generalizations. Next, we introduce another constant, usually referred to as the Piterbarg constant, given by

$$
\mathcal{P}_{\alpha}^{a}:=\lim _{S \rightarrow \infty} \mathcal{P}_{\alpha}^{a}[0, S] \in(0, \infty), \quad \alpha \in(0,2], a>0,
$$

where

$$
\mathcal{P}_{\alpha}^{a}[S, T]=\mathbb{E}\left(\exp \left(\sup _{t \in[S, T]}\left(\sqrt{2} B_{\alpha}(t)-(1+a)|t|^{\alpha}\right)\right)\right), \quad 0 \leq S<T<\infty .
$$

It is also known that

$$
\mathcal{P}_{1}^{a}=1+\frac{1}{a} \quad \text { and } \quad \mathcal{P}_{2}^{a}=\frac{1}{2}\left(1+\sqrt{1+\frac{1}{a}}\right)
$$

see, e.g. Dębicki and Mandjes (2003) and Dębicki and Tabiś (2011). As will be seen in Theorem 2.1 below, both Pickands' constant and Piterbarg's constant are important for our study. We denote by $\Phi(\cdot)$ the standard normal distribution (of an $N(0,1)$ random variable), and, furthermore, set $\Psi(\cdot):=1-\Phi(\cdot)$.

In the following we investigate the tail asymptotic behavior of the supremum of nonstationary Gaussian random fields over a region which depends on $u$. Our next result is of interest on its own, and, furthermore, is the key to the proof of Theorem 1.1.

Theorem 2.1. Let $S$ and $T$ be two positive constants, and let $\{X(s, t),(s, t) \in[0, S] \times[0, T]\}$ be a centered Gaussian random field, with standard deviation function $\sigma(\cdot, \cdot)$ and correlation function $r(\cdot, \cdot, \cdot, \cdot)$. Assume that $\sigma(\cdot, \cdot)$ attains its maximum on $[0, S] \times[0, T]$ at the unique point $\left(0, t_{0}\right)$ with $t_{0} \in(0, T)$, and, furthermore, that

$$
\sigma(s, t)=1-b_{1} s^{\beta}(1+o(1))-b_{2}\left|t-t_{0}\right|^{2}(1+o(1))-b_{3} s\left|t-t_{0}\right|(1+o(1))
$$

as $(s, t) \rightarrow\left(0, t_{0}\right)$ for some constants $\beta \in(1,2), b_{i}>0, i=1,2$, and $b_{3} \in \mathbb{R}$ satisfying $b_{2}+b_{3} / 2>0$. Suppose, furthermore, that

$$
r\left(s, s^{\prime}, t, t^{\prime}\right)=1-\left(a_{1}\left|s-s^{\prime}\right|^{\beta}+a_{2}\left|t-t^{\prime}\right|^{\beta}\right)(1+o(1)) \quad \text { as }(s, t),\left(s^{\prime}, t^{\prime}\right) \rightarrow\left(0, t_{0}\right)
$$

for some constants $a_{i}>0, i=1,2$. Then, for any $x \in \mathbb{R}$,

$$
\begin{aligned}
& \mathbb{P}\left\{\sup _{(s, t) \in \widetilde{\Delta}_{x}^{1}(u)} X(s, t)>u\right\}=\sqrt{\frac{\pi}{b_{2}}} a_{2}^{1 / \beta} \mathcal{P}_{\beta}^{b_{1} / a_{1}} \mathscr{H}_{\beta} u^{2 / \beta-1} \Psi(u) \Phi\left(\sqrt{2 b_{2}} x\right)(1+o(1)), \\
& \mathbb{P}\left\{\sup _{(s, t) \in \triangle_{x}^{2}(u)} X(s, t)>u\right\}=\sqrt{\frac{\pi}{b_{2}}} a_{2}^{1 / \beta} \mathcal{P}_{\beta}^{b_{1} / a_{1}} \mathscr{H}_{\beta} u^{2 / \beta-1} \Psi(u) \Psi\left(\sqrt{2 b_{2}} x\right)(1+o(1)),
\end{aligned}
$$


as $u \rightarrow \infty$, where $\delta_{1}(u)=(\ln u / u)^{2 / \beta}, \delta_{2}(u)=\ln u / u$, and

$\widetilde{\Delta_{x}^{1}}(u)=\left[0, \delta_{1}(u)\right] \times\left[t_{0}-\delta_{2}(u), t_{0}+x u^{-1}\right], \quad \widetilde{\Delta_{x}^{2}}(u)=\left[0, \delta_{1}(u)\right] \times\left[t_{0}+x u^{-1}, t_{0}+\delta_{2}(u)\right]$.

Remarks. (a) If $\beta \in(0,1)$ then (2.1) becomes

$$
\sigma(s, t)=1-b_{1} s^{\beta}(1+o(1))-b_{2}\left|t-t_{0}\right|^{2}(1+o(1)) \quad \text { as }(s, t) \rightarrow\left(0, t_{0}\right) .
$$

We mention that in this case both (2.3) and (2.4) are still valid.

(b) It can be shown along the lines of the proof of Theorem 2.1 that if $x=x(u)$ satisfies the conditions

$$
\lim _{u \rightarrow \infty} x(u)=\infty, \quad x(u)=o\left(u^{\varepsilon}\right), \quad \text { as } u \rightarrow \infty \text { for any } \varepsilon>0,
$$

then (2.3) still holds with $\Phi\left(\sqrt{2 b_{2}} x\right)$ replaced by 1 . Similarly, if $x=-x(u)$ with $x(u)$ satisfying (2.5) then (2.4) holds with $\Psi\left(\sqrt{2 b_{2}} x\right)$ replaced by 1 .

\subsection{Proof of Theorem 1.1}

Define

$$
T_{1}(u)=\inf \left\{t \geq 0: Y(s, t)>u^{1-H} \text { for some } s \in[0, t]\right\}
$$

and

$$
T_{2}(u)=\sup \left\{t \geq 0: Y(s, t)>u^{1-H} \text { for some } s \in[0, t]\right\} .
$$

Clearly, $\tau_{i}(u) \stackrel{\mathrm{D}}{=} u T_{i}(u), i=1,2$. Consider first the approximation of $\tau_{1}(u)$. For any $x \in \mathbb{R}$ and $u>0$, we have

$$
\begin{aligned}
\mathbb{P}\left\{\frac{\tau_{1}(u)-\tilde{t}_{0} u}{A(u)} \leq x \mid \tau_{1}(u)<\infty\right\} & =\mathbb{P}\left\{T_{1}(u) \leq \tilde{t}_{0}+x A(u) u^{-1} \mid T_{1}(u)<\infty\right\} \\
& =\frac{\mathbb{P}\left\{\sup _{0 \leq s \leq t \leq \tilde{t}_{0}+x A(u) u^{-1}} Y(s, t)>u^{1-H}\right\}}{\mathbb{P}\left\{\tau_{1}(u)<\infty\right\}}
\end{aligned}
$$

In view of Hashorva et al. (2013), for any $H, \gamma \in(0,1)$,

$$
\mathbb{P}\left\{\tau_{1}(u)<\infty\right\}=\mathbb{P}\left\{\sup _{t \geq 0} W_{\gamma}(t)>u\right\}=W_{H}(u) \Psi\left(\frac{c^{H} u^{1-H}}{H^{H}(1-H)^{1-H}}\right)(1+o(1))
$$

as $u \rightarrow \infty$, where

$$
\mathcal{W}_{H}(u)=2^{1 / 2-1 / 2 H} \frac{\sqrt{\pi}}{\sqrt{H(1-H)}} \mathscr{H}_{2 H} \mathcal{P}_{2 H}^{(1-\gamma) / \gamma}\left(\frac{c^{H} u^{1-H}}{H^{H}(1-H)^{1-H}}\right)^{1 / H-1} .
$$

Next, we focus on the analysis of $\mathbb{P}\left\{\sup _{0 \leq s \leq t \leq \tilde{t}_{0}+x A(u) u^{-1}} Y(s, t)>u^{1-H}\right\}$. By Bonforroni's inequality,

$$
p_{3}(u) \leq \mathbb{P}\left\{\sup _{0 \leq s \leq t \leq \tilde{t}_{0}+x A(u) u^{-1}} Y(s, t)>u^{1-H}\right\} \leq p_{1}(u)+p_{2}(u)+p_{3}(u),
$$

where $p_{i}(u), i=1,2,3$, are defined in (2.8), (2.12), and (2.13) below. In the following we will derive the asymptotics of $p_{3}(u)$ as $u \rightarrow \infty$, and give bounds for both $p_{1}(u)$ and $p_{2}(u)$ for large $u$, assuring that they are relatively negligible. 
We first consider bounds for $p_{1}(u)$ and $p_{2}(u)$. Since on the set $\{(s, t): 0 \leq s \leq t<\infty\}$ the maximum of the variance function $V_{Y}^{2}(s, t)$ is attained uniquely at $\left(0, \tilde{t}_{0}\right)$, we obtain from the Borell-TIS inequality (see, e.g. Adler and Taylor (2007)) that, for any constant $K \geq 2 \tilde{t}_{0}$, there exist constants $\rho>0$ small enough and $\theta \in(0,1)$ such that, for sufficiently large $u$,

$$
p_{1}(u):=\mathbb{P}\left\{\sup _{\substack{0 \leq s \leq t \leq K \\ s \in[\rho, K] \text { or } t \in\left[0, \tilde{t}_{0}-\rho\right]}} Y(s, t)>u^{1-H}\right\} \leq \exp \left(-\frac{\left(u^{1-H}-d\right)^{2}}{2 \theta V_{Y}^{2}\left(0, \tilde{t}_{0}\right)}\right),
$$

with $d=\mathbb{E}\left(\sup _{0 \leq s \leq t \leq K} Y(s, t)\right)<\infty$. It follows that

$$
1-\frac{V_{Y}(s, t)}{V_{Y}\left(0, \tilde{t}_{0}\right)}= \begin{cases}\frac{c^{2}(1-H)^{3}}{2 H}\left(\tilde{t}_{0}-t\right)^{2}(1+o(1)) & \\ +\frac{\left(\gamma-\gamma^{2}\right)(1-H)^{2 H} c^{2 H}}{2 H^{2 H}} s^{2 H}(1+o(1)), & H \leq \frac{1}{2}, \\ \frac{c^{2}(1-H)^{3}}{2 H}\left(\tilde{t}_{0}-t+\gamma s\right)^{2}(1+o(1)) & \\ +\frac{\left(\gamma-\gamma^{2}\right)(1-H)^{2 H} c^{2 H}}{2 H^{2 H}} s^{2 H}(1+o(1)), & H>\frac{1}{2},\end{cases}
$$

as $(s, t) \rightarrow\left(0, \tilde{t}_{0}\right)$, and, furthermore, the correlation function of $Y$ satisfies

$$
1-\operatorname{cov}\left(\frac{Y(s, t)}{V_{Y}(s, t)}, \frac{Y\left(s^{\prime}, t^{\prime}\right)}{V_{Y}\left(s^{\prime}, t^{\prime}\right)}\right)=\frac{1}{2 \tilde{t}_{0}^{2 H}}\left(\left|t-t^{\prime}\right|^{2 H}+\gamma^{2}\left|s-s^{\prime}\right|^{2 H}\right)(1+o(1))
$$

as $(s, t),\left(s^{\prime}, t^{\prime}\right) \rightarrow\left(0, \tilde{t}_{0}\right)$. In addition, for $\rho>0$ chosen small enough, there exists some $Q>0$ such that, for any $(s, t),\left(s^{\prime}, t^{\prime}\right) \in[0, \rho] \times\left[\tilde{t}_{0}-\rho, \tilde{t}_{0}+\rho\right]$,

$$
\mathbb{E}\left(Y(s, t)-Y\left(s^{\prime}, t^{\prime}\right)\right)^{2} \leq Q\left(\left|t-t^{\prime}\right|^{2 H}+\left|s-s^{\prime}\right|^{2 H}\right) .
$$

Next, let

$$
A=\frac{H^{1 / 2}}{c(1-H)^{3 / 2}}, \quad \tilde{u}=\frac{u^{1-H}}{V_{Y}\left(0, \tilde{t}_{0}\right)} .
$$

In light of (2.9) and (2.11), by the Piterbarg inequality (see Theorem 8.1 of Piterbarg (1996) or Theorem 8.1 of Piterbarg (2001)), for all sufficiently large $u$,

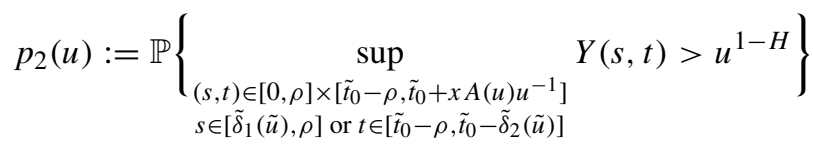

$$
\begin{aligned}
& \leq C_{1} u^{2(1-H) / H} \exp \left(-\frac{u^{2(1-H)}}{2 V_{Y}^{2}\left(0, \tilde{t}_{0}\right)}-C_{2}(\ln u)^{2}\right)
\end{aligned}
$$

for some positive constants $C_{i}, i=1,2$, where $\tilde{\delta}_{1}(\tilde{u})=(\ln \tilde{u} / \tilde{u})^{1 / H}$ and $\tilde{\delta}_{2}(\tilde{u})=\ln \tilde{u} / \tilde{u}$. Furthermore, we have

$$
\begin{aligned}
& p_{3}(u):=\mathbb{P}\left\{\sup _{(s, t) \in\left[0, \tilde{\delta}_{1}(\tilde{u})\right] \times\left[\tilde{t}_{0}-\tilde{\delta}_{2}(\tilde{u}), \tilde{t}_{0}+x A(u) u^{-1}\right]} Y(s, t)>u^{1-H}\right\} \\
& =\mathbb{P}\left\{\sup _{(s, t) \in \widehat{\Delta_{A x}^{1}}(\tilde{u})} \frac{Y(s, t)}{V_{Y}\left(0, \tilde{t}_{0}\right)}>\tilde{u}\right\},
\end{aligned}
$$


where $\widehat{\Delta_{A x}^{1}}(\tilde{u})=\left[0, \tilde{\delta}_{1}(\tilde{u})\right] \times\left[\tilde{t}_{0}-\tilde{\delta}_{2}(\tilde{u}), \tilde{t}_{0}+A x \tilde{u}^{-1}\right]$. Utilizing (2.9) and (2.10), it follows from Theorem 2.1 that

$$
\begin{aligned}
& \mathbb{P}\left\{\sup _{(s, t) \in \widehat{\Delta_{A x}^{1}}(\tilde{u})} \frac{Y(s, t)}{V_{Y}\left(0, \tilde{t}_{0}\right)}>\tilde{u}\right\} \\
& =\mathscr{H}_{2 H} \mathcal{P}_{2 H}^{(1-\gamma) / \gamma} 2^{-1 / 2 H} \sqrt{2 \pi} A \frac{c(1-H)}{H} \Psi(\tilde{u}) \tilde{u}^{1 / H-1} \Phi(x)(1+o(1))
\end{aligned}
$$

as $u \rightarrow \infty$. Consequently, we conclude from (2.7)-(2.8) and (2.12)-(2.14) that

$$
\begin{aligned}
\mathbb{P}\left\{\sup _{0 \leq s \leq t \leq \tilde{t}_{0}+x A(u) u^{-1}} Y(s, t)>u^{1-H}\right\} \\
=\mathscr{H}_{2 H} \mathcal{P}_{2 H}^{(1-\gamma) / \gamma} 2^{-1 / 2 H} \sqrt{2 \pi} A \frac{c(1-H)}{H} \Psi(\tilde{u}) \tilde{u}^{1 / H-1} \Phi(x)(1+o(1))
\end{aligned}
$$

as $u \rightarrow \infty$, and, thus, in light of (2.6),

$$
\lim _{u \rightarrow \infty} \sup _{x \in \mathbb{R}}\left|\mathbb{P}\left\{\frac{\tau_{1}(u)-\tilde{t}_{0} u}{A(u)} \leq x \mid \tau_{1}(u)<\infty\right\}-\Phi(x)\right|=0 .
$$

Using similar arguments, we conclude from the properties of the random field $Y$ and (2.4) that

$$
\begin{aligned}
& \mathbb{P}\left\{\sup _{t \geq \tilde{t}_{0}+x A(u) u^{-1}, s \in[0, t]} Y(s, t)>u^{1-H}\right\} \\
& =\mathscr{H}_{2 H} \mathcal{P}_{2 H}^{(1-\gamma) / \gamma} 2^{-1 / 2 H} \sqrt{2 \pi} A \frac{c(1-H)}{H} \Psi(\tilde{u}) \tilde{u}^{1 / H-1} \Psi(x)(1+o(1))
\end{aligned}
$$

as $u \rightarrow \infty$, where we used the fact that, for any large enough integer $K>\tilde{t}_{0}$,

$$
\mathbb{P}\left\{\sup _{0 \leq s \leq t<\infty} Y(s, t)>u^{1-H}\right\}=\mathbb{P}\left\{\sup _{0 \leq s \leq t<K} Y(s, t)>u^{1-H}\right\}(1+o(1)) \quad \text { as } u \rightarrow \infty ;
$$

see Hashorva et al. (2013). Therefore,

$$
\begin{aligned}
\mathbb{P}\left\{\frac{\tau_{2}(u)-\tilde{t}_{0} u}{A(u)} \leq x \mid \tau_{1}(u)<\infty\right\} & =1-\mathbb{P}\left\{\frac{\tau_{2}(u)-\tilde{t}_{0} u}{A(u)} \geq x \mid \tau_{1}(u)<\infty\right\} \\
& =1-\mathbb{P}\left\{T_{2}(u) \geq \tilde{t}_{0}+x A(u) u^{-1} \mid T_{1}(u)<\infty\right\} \\
& =1-\frac{\mathbb{P}\left\{\sup _{t \geq \tilde{t}_{0}+x A(u) u^{-1}, s \in[0, t]} Y(s, t)>u^{1-H}\right\}}{\mathbb{P}\left\{\tau_{1}(u)<\infty\right\}} \\
& \rightarrow \Phi(x) \text { as } u \rightarrow \infty
\end{aligned}
$$

for any $x \in \mathbb{R}$. Hence, the proof follows by a direct application of Lemma 2.1 below.

Lemma 2.1. Let $\left(Z_{u 1}, Z_{u 2}\right), u>0$, be a bivariate random sequence such that $Z_{u 2} \geq Z_{u 1}$ almost surely for all large $u$. If the convergence in distribution

$$
Z_{u i} \stackrel{\mathrm{D}}{\rightarrow} \mathrm{Z} \quad \text { as } u \rightarrow \infty
$$

holds for $i=1,2$ with $\mathcal{Z}$ a nondegenerate random variable, then we have the joint convergence in distribution

$$
\left(Z_{u 1}, Z_{u 2}\right) \stackrel{\mathrm{D}}{\rightarrow}(\mathcal{Z}, \mathcal{Z}) \quad \text { as } u \rightarrow \infty
$$


Proof. Let $x$ and $y$ be any two continuous points of the distribution function $\mathbb{P}\{\mathcal{Z} \leq t\}$, $t \in \mathbb{R}$. It is sufficient to show that

$$
\lim _{u \rightarrow \infty} \mathbb{P}\left\{Z_{u 1} \leq x, Z_{u 2} \leq y\right\}=\mathbb{P}\{\mathcal{Z} \leq \min (x, y)\} .
$$

In fact, if $x \geq y$ by the assumption that $Z_{u 2} \geq Z_{u 1}$ holds for all large $u$, we have

$$
\mathbb{P}\left\{Z_{u 1} \leq x, Z_{u 2} \leq y\right\}=\mathbb{P}\left\{Z_{u 2} \leq y\right\} \rightarrow \mathbb{P}\{\mathcal{Z} \leq y\} \quad \text { as } u \rightarrow \infty .
$$

Furthermore, if $x \leq y$,

$$
\begin{aligned}
\mathbb{P}\left\{Z_{u 1} \leq x, Z_{u 2} \leq y\right\} & =\mathbb{P}\left\{Z_{u 1} \leq x\right\}-\mathbb{P}\left\{Z_{u 1} \leq x, Z_{u 2}>y\right\} \\
& \geq \mathbb{P}\left\{Z_{u 1} \leq x\right\}-\mathbb{P}\left\{Z_{u 1} \leq y, Z_{u 2}>y\right\} \\
& =\mathbb{P}\left\{Z_{u 1} \leq x\right\}-\left(\mathbb{P}\left\{Z_{u 2}>y\right\}-\mathbb{P}\left\{Z_{u 1}>y\right\}\right) \\
& \rightarrow \mathbb{P}\{\mathcal{Z} \leq x\} \quad \text { as } u \rightarrow \infty
\end{aligned}
$$

and

$$
\mathbb{P}\left\{Z_{u 1} \leq x, Z_{u 2} \leq y\right\} \leq \mathbb{P}\left\{Z_{u 1} \leq x\right\} \rightarrow \mathbb{P}\{\mathcal{Z} \leq x\} \quad \text { as } u \rightarrow \infty
$$

hold; hence, the claim follows.

\section{Proof of Theorem 2.1}

We present only the proof of (2.3) with $x \geq 0$, since the other cases can be dealt with similarly. For simplicity, we will assume that $a_{1}=a_{2}=1$; the general case follows by a time scaling.

Since our approach is asymptotic in nature, and both $\delta_{1}(u)$ and $\delta_{2}(u)$ converge to 0 as $u$ tends to $\infty$, properties (2.1) and (2.2) are the only necessary properties of the Gaussian random field $X$ needed for the asymptotics (which can be seen from the proof below). Therefore, we conclude that

$$
\mathbb{P}\left\{\sup _{(s, t) \in \widetilde{\Delta_{x}^{1}}(u)} X(s, t)>u\right\}=\mathbb{P}\left\{\sup _{(s, t) \in \widetilde{\Delta}_{x}^{1}(u)} \tilde{\xi}(s, t)>u\right\}(1+o(1))=: \pi(u)(1+o(1))
$$

as $u \rightarrow \infty$, with $\{\tilde{\xi}(s, t), s, t \geq 0\}$ any Gaussian random field possessing properties (2.1) and (2.2). In particular, we choose

$$
\tilde{\xi}(s, t)=\frac{\xi(s, t)}{\left(1+b_{1} s^{\beta}\right)\left(1+b_{2}\left|t-t_{0}\right|^{2}+b_{3}\left|t-t_{0}\right| s\right)}, \quad s, t \geq 0,
$$

with $\{\xi(s, t), s, t \in \mathbb{R}\}$ a centered homogeneous Gaussian random field with covariance function

$$
r_{\xi}(s, t)=\exp \left(-|s|^{\beta}-|t|^{\beta}\right), \quad s, t \in \mathbb{R} .
$$

Since $\beta<2$, for any positive constants $S_{1}$ and $S_{2}$, we can divide the intervals $\left[0, \delta_{1}(u)\right]$ and $\left[t_{0}-\delta_{2}(u), t_{0}+x u^{-1}\right]$ into several subintervals of length $S_{1} u^{-2 / \beta}$ and $S_{2} u^{-2 / \beta}$, respectively. Specifically, for $S_{1}, S_{2}>0$, let

$$
\triangle_{0}^{i}=u^{-2 / \beta}\left[0, S_{i}\right], \quad \triangle_{k}^{i}=u^{-2 / \beta}\left[k S_{i},(k+1) S_{i}\right], \quad k \in \mathbb{Z}, i=1,2 .
$$


Furthermore, for any $u>0$, let

$$
\begin{gathered}
h_{1}(u)=\left\lfloor S_{1}^{-1}(\ln u)^{2 / \beta}\right\rfloor+1, \quad h_{2}(u)=\left\lfloor S_{2}^{-1}(\ln u) u^{2 / \beta-1}\right\rfloor+1, \\
h_{2, x}(u)=\left\lfloor S_{2}^{-1} x u^{2 / \beta-1}\right\rfloor+1 .
\end{gathered}
$$

Here $\lfloor\cdot\rfloor$ denotes the ceiling function. Applying Bonferroni's inequality we obtain

$$
\begin{aligned}
\pi(u) \leq & \sum_{k_{1}=0}^{h_{1}(u)} \sum_{k_{2}=-h_{2}(u)}^{h_{2, x}(u)} \mathbb{P}\left\{\sup _{(s, t) \in \Delta_{k_{1}}^{1} \times\left(t_{0}+\triangle_{k_{2}}^{2}\right)} \tilde{\xi}(s, t)>u\right\} \\
= & \sum_{k_{2}=-h_{2}(u)}^{h_{2, x}(u)} \mathbb{P}\left\{\sup _{(s, t) \in \Delta_{0}^{1} \times\left(t_{0}+\triangle_{k_{2}}^{2}\right)} \tilde{\xi}(s, t)>u\right\} \\
& +\sum_{k_{1}=1}^{h_{1}(u)} \sum_{k_{2}=-h_{2}(u)}^{h_{2, x}(u)} \mathbb{P}\left\{\sup _{(s, t) \in \triangle_{k_{1}}^{1} \times\left(t_{0}+\triangle_{k_{2}}^{2}\right)} \tilde{\xi}(s, t)>u\right\} \\
= & : I_{1, x}(u)+I_{2, x}(u)
\end{aligned}
$$

and

$$
\pi(u) \geq J_{1, x}(u)-J_{2, x}(u),
$$

where

$$
\begin{aligned}
& J_{1, x}(u)= \sum_{k_{2}=-h_{2}(u)+1}^{h_{2, x}(u)-1} \mathbb{P}\left\{\sup _{(s, t) \in \Delta_{0}^{1} \times\left(t_{0}+\triangle_{k_{2}}^{2}\right)} \tilde{\xi}(s, t)>u\right\}, \\
& J_{2, x}(u)= \sum_{-h_{2}(u)+1 \leq i<j \leq h_{2, x}(u)-1} \mathbb{P}\left\{\sup _{(s, t) \in \Delta_{0}^{1} \times\left(t_{0}+\triangle_{i}^{2}\right)} \tilde{\xi}(s, t)>u,\right. \\
&\left.\sup _{(s, t) \in \triangle_{0}^{1} \times\left(t_{0}+\triangle_{j}^{2}\right)} \tilde{\xi}(s, t)>u\right\} .
\end{aligned}
$$

Next, we derive the required asymptotic bounds of $I_{1, x}(u)$ and $J_{1, x}(u)$, and show that

$$
I_{2, x}(u)=J_{2, x}(u)(1+o(1))=o\left(I_{1, x}(u)\right)=o\left(J_{1, x}(u)\right)
$$

as $u \rightarrow \infty$ and $S_{i} \rightarrow \infty, i=1,2$. Assuming, furthermore, that $b_{3}>0$, we have

$$
\begin{aligned}
J_{1, x}(u) \geq & \sum_{k_{2}=0}^{h_{2, x}(u)-1} \mathbb{P}\left\{\sup _{(s, t) \in \triangle_{0}^{1} \times \triangle_{k_{2}}^{2}} \frac{\xi(s, t)}{1+b_{1} s^{\beta}}>u\left(1+b_{2}\left(\left(k_{2}+1\right) S_{2} u^{-2 / \beta}\right)^{2}\right.\right. \\
& \left.\left.+b_{3}\left(\left(k_{2}+1\right) S_{2} u^{-2 / \beta}\right)\left(S_{1} u^{-2 / \beta}\right)\right)\right\}
\end{aligned}
$$


In view of Lemma A.1 in Appendix A,

$$
\begin{aligned}
J_{1,1, x}(u)= & (1+o(1)) \mathcal{P}_{\beta}^{b_{1}}\left[0, S_{1}\right] \mathcal{H}_{\beta}\left[0, S_{2}\right] \frac{1}{\sqrt{2 \pi} u} \\
& \times \sum_{k_{2}=0}^{h_{2, x}(u)-1} \frac{1}{1+b_{2}\left(\left(k_{2}+1\right) S_{2} u^{-2 / \beta}\right)^{2}+b_{3}\left(\left(k_{2}+1\right) S_{2} u^{-2 / \beta}\right) S_{1} u^{-2 / \beta}} \\
& \times \exp \left(-\frac{u^{2}\left(1+b_{2}\left(\left(k_{2}+1\right) S_{2} u^{-2 / \beta}\right)^{2}+b_{3}\left(\left(k_{2}+1\right) S_{2} u^{-2 / \beta}\right) S_{1} u^{-2 / \beta}\right)^{2}}{2}\right) \\
= & \mathcal{P}_{\beta}^{b_{1}}\left[0, S_{1}\right] \mathscr{H}_{\beta}\left[0, S_{2}\right] \Psi(u)(1+o(1)) \\
& \times \sum_{k_{2}=0}^{h_{2, x}(u)-1} \exp \left(-b_{2}\left(\left(k_{2}+1\right) S_{2} u^{1-2 / \beta}\right)^{2}-b_{3} u^{2}\left(\left(k_{2}+1\right) S_{2} u^{-2 / \beta}\right) S_{1} u^{-2 / \beta}\right) \\
= & \mathcal{P}_{\beta}^{b_{1}}\left[0, S_{1}\right] \frac{\mathcal{H}_{\beta}\left[0, S_{2}\right]}{S_{2}} \Psi(u) u^{2 / \beta-1} \int_{0}^{x} \mathrm{e}^{-b_{2} y^{2}} \mathrm{~d} y(1+o(1))
\end{aligned}
$$

as $u \rightarrow \infty$, where in the last equation we utilized the facts that

$$
h_{2, x}(u) \rightarrow \infty, \quad h_{2, x}(u) S_{2} u^{1-2 / \beta} \rightarrow x, \quad u^{2}\left(h_{2, x}(u) S_{2} u^{-2 / \beta}\right)\left(S_{1} u^{-2 / \beta}\right) \rightarrow 0,
$$

as $u \rightarrow \infty$. Similarly,

$$
J_{1,2, x}(u)=\mathcal{P}_{\beta}^{b_{1}}\left[0, S_{1}\right] \frac{\mathcal{H}_{\beta}\left[0, S_{2}\right]}{S_{2}} \Psi(u) u^{2 / \beta-1} \int_{-\infty}^{0} \mathrm{e}^{-b_{2} y^{2}} \mathrm{~d} y(1+o(1))
$$

as $u \rightarrow \infty$. Therefore, we conclude that

$$
J_{1, x}(u) \geq \mathcal{P}_{\beta}^{b_{1}}\left[0, S_{1}\right] \frac{\mathcal{H}_{\beta}\left[0, S_{2}\right]}{S_{2}} \Psi(u) u^{2 / \beta-1} \int_{-\infty}^{x} \mathrm{e}^{-b_{2} y^{2}} \mathrm{~d} y(1+o(1))
$$

as $u \rightarrow \infty$. Using similar arguments, we further obtain

$$
\begin{aligned}
I_{1, x}(u) \leq & \sum_{k_{2}=0}^{h_{2, x}(u)-1} \mathbb{P}\left\{\sup _{(s, t) \in \Delta_{0}^{1} \times \Delta_{k_{2}}^{2}} \frac{\xi(s, t)}{1+b_{1} s^{\beta}}>u\left(1+b_{2}\left(k_{2} S_{2} u^{-2 / \beta}\right)^{2}\right)\right\} \\
& +\sum_{k_{2}=-h_{2}(u)} \mathbb{P}\left\{\sup _{(s, t) \in \triangle_{0}^{1} \times \triangle_{k_{2}}^{2}} \frac{\xi(s, t)}{1+b_{1} s^{\beta}}>u\left(1+b_{2}\left(-\left(k_{2}+1\right) S_{2} u^{-2 / \beta}\right)^{2}\right)\right\} \\
= & \mathcal{P}_{\beta}^{b_{1}}\left[0, S_{1}\right] \frac{\mathcal{H}_{\beta}\left[0, S_{2}\right]}{S_{2}} \Psi(u) u^{2 / \beta-1} \int_{-\infty}^{x} \mathrm{e}^{-b_{2} y^{2}} \mathrm{~d} y(1+o(1))
\end{aligned}
$$


as $u \rightarrow \infty$. Next we verify (3.1). Specifically,

$$
\begin{aligned}
I_{2, x}(u) \leq & \sum_{k_{1}=1}^{h_{1}(u)} \sum_{k_{2}=0}^{h_{2, x}(u)} \mathbb{P}\left\{\sup _{(s, t) \in \triangle_{k_{1}}^{1} \times \triangle_{k_{2}}^{2}} \xi(s, t)>u\left(1+b_{1}\left(k_{1} S_{1} u^{-2 / \beta}\right)^{\beta}+b_{2}\left(k_{2} S_{2} u^{-2 / \beta}\right)^{2}\right)\right\} \\
& +\sum_{k_{1}=1}^{h_{1}(u)} \sum_{k_{2}=-h_{2}(u)}^{-1} \mathbb{P}\left\{\sup _{(s, t) \in \triangle_{k_{1}}^{1} \times \triangle_{k_{2}}^{2}} \xi(s, t)>u\left(1+b_{1}\left(k_{1} S_{1} u^{-2 / \beta}\right)^{\beta}\right.\right. \\
& \left.\left.+b_{2}\left(-\left(k_{2}+1\right) S_{2} u^{-2 / \beta}\right)^{2}\right)\right\} .
\end{aligned}
$$

Applying similar arguments as in (3.4) yield

$$
\begin{aligned}
I_{2, x}(u) \leq & \mathcal{H}_{\beta}\left[0, S_{1}\right] \mathcal{H}_{\beta}\left[0, S_{2}\right] \Psi(u)\left(S_{2}^{-1} u^{2 / \beta-1}\right)(1+o(1)) \\
& \times \int_{-\infty}^{x} \mathrm{e}^{-b_{2} y^{2}} \mathrm{~d} y \sum_{k_{1}=1}^{h_{1}(u)} \exp \left(-b_{1}\left(k_{1} S_{1}\right)^{\beta}\right)
\end{aligned}
$$

as $u \rightarrow \infty$. Furthermore, we write

$$
\begin{aligned}
J_{2, x}(u) & =\sum_{-h_{2}(u)+1 \leq i<j \leq h_{2, x}(u)-1} \mathbb{P}\left\{\sup _{(s, t) \in \triangle_{0}^{1} \times\left(t_{0}+\triangle_{i}^{2}\right)} \tilde{\xi}(s, t)>u, \sup _{(s, t) \in \Delta_{0}^{1} \times\left(t_{0}+\triangle_{j}^{2}\right)} \tilde{\xi}(s, t)>u\right\} \\
& =: \Sigma_{1, x}(u)+\Sigma_{2, x}(u),
\end{aligned}
$$

where $\Sigma_{1, x}(u)$ is the sum over indexes $j=i+1$, and $\Sigma_{2, x}(u)$ is the sum over indexes $j>i+1$. Let $B\left(i, S_{2}, u\right)=u\left(1+b_{2}\left(|i| S_{2} u^{-2 / \beta}\right)^{2}\right), i \in \mathbb{Z}, S_{2}>0, u>0$. It follows that

$$
\begin{aligned}
& \Sigma_{1, x}(u) \leq \sum_{i=-1}^{h_{2, x}(u)-1} \mathbb{P}\left\{\sup _{(s, t) \in \Delta_{0}^{1} \times \triangle_{i}^{2}} \frac{\xi(s, t)}{1+b_{1} s^{\beta}}>B\left(0, S_{2}, u\right),\right. \\
&\left.+\sum_{i=-h_{2}(u)+1} \sup _{(s, t) \in \Delta_{0}^{1} \times \triangle_{i+1}^{2}} \frac{\xi(s, t)}{1+b_{1} s^{\beta}}>B\left(0, S_{2}, u\right)\right\} \\
& \sup _{(s, t) \in \Delta_{0}^{1} \times \triangle_{i}^{2}} \frac{\xi(s, t)}{1+b_{1} s^{\beta}}>B\left(i+2, S_{2}, u\right), \\
&\left.\sup _{(s, t) \in \triangle_{0}^{1} \times \triangle_{i+1}^{2}} \frac{\xi(s, t)}{1+b_{1} s^{\beta}}>B\left(i+2, S_{2}, u\right)\right\}
\end{aligned}
$$

and, for any $i, j \in \mathbb{Z}$,

$$
\begin{aligned}
& \mathbb{P}\left\{\sup _{(s, t) \in \Delta_{0}^{1} \times \triangle_{i}^{2}} \frac{\xi(s, t)}{1+b_{1} s^{\beta}}>B\left(j, S_{2}, u\right), \sup _{(s, t) \in \Delta_{0}^{1} \times \triangle_{i+1}^{2}} \frac{\xi(s, t)}{1+b_{1} s^{\beta}}>B\left(j, S_{2}, u\right)\right\} \\
&= \mathbb{P}\left\{\sup _{(s, t) \in \triangle_{0}^{1} \times \triangle_{0}^{2}} \frac{\xi(s, t)}{1+b_{1} s^{\beta}}>B\left(j, S_{2}, u\right)\right\}+\mathbb{P}\left\{\sup _{(s, t) \in \Delta_{0}^{1} \times \triangle_{1}^{2}} \frac{\xi(s, t)}{1+b_{1} s^{\beta}}>B\left(j, S_{2}, u\right)\right\} \\
&-\mathbb{P}\left\{\sup _{(s, t) \in \Delta_{0}^{1} \times\left(\triangle_{0}^{2} \cup \triangle_{1}^{2}\right)} \frac{\xi(s, t)}{1+b_{1} s^{\beta}}>B\left(j, S_{2}, u\right)\right\} .
\end{aligned}
$$


Therefore, analogous to the derivation of (3.4), we obtain

$$
\limsup _{u \rightarrow \infty} \frac{\Sigma_{1, x}(u)}{\Psi(u) u^{2 / \beta-1}} \leq \mathcal{P}_{\beta}^{b_{1}}\left[0, S_{1}\right] \frac{2 \mathscr{H}_{\beta}\left[0, S_{2}\right]-\mathscr{H}_{\beta}\left[0,2 S_{2}\right]}{S_{2}}\left(x+\int_{-\infty}^{0} \mathrm{e}^{-b_{2} y^{2}} \mathrm{~d} y\right) .
$$

Furthermore, for any $u>0$,

$$
\begin{aligned}
& \Sigma_{2, x}(u) \leq \sum_{i=-1}^{h_{2, x}(u)-1} \sum_{j \geq 2} \mathbb{P}\left\{\sup _{(s, t) \in \triangle_{0}^{1} \times \triangle_{0}^{2}} \xi(s, t)>u, \sup _{(s, t) \in \triangle_{0}^{1} \times \triangle_{j}^{2}} \xi(s, t)>u\right\} \\
& +\sum_{i=-h_{2}(u)+1}^{-2} \sum_{j \geq 2} \mathbb{P}\left\{\sup _{(s, t) \in \triangle_{0}^{1} \times \triangle_{0}^{2}} \xi(s, t)>B\left(i+1, S_{2}, u\right), \sup _{(s, t) \in \triangle_{0}^{1} \times \triangle_{j}^{2}} \xi(s, t)>u\right\} \\
& \leq \sum_{i=-1}^{h_{2, x}(u)-1} \sum_{j \geq 2} \mathbb{P}\left\{\sup _{\substack{\left(s^{\prime}, t^{\prime}\right) \in \triangle_{0}^{1} \times \triangle_{j}^{2} \\
(s, t) \in \triangle_{0}^{1} \times \triangle_{0}^{2}}} \zeta\left(s, t, s^{\prime}, t^{\prime}\right)>2 u\right\} \\
& +\sum_{i=-h_{2}(u)+1}^{-2} \sum_{j \geq 2} \mathbb{P}\left\{\sup _{\substack{\left(s^{\prime}, t^{\prime}\right) \in \Delta_{0}^{1} \times \triangle_{j}^{2} \\
(s, t) \in \triangle_{0}^{1} \times \triangle_{0}^{2}}} \zeta\left(s, t, s^{\prime}, t^{\prime}\right)>B\left(i+1, s_{2}, u\right)+u\right\},
\end{aligned}
$$

where

$$
\zeta\left(s, t, s^{\prime}, t^{\prime}\right)=\xi(s, t)+\xi\left(s^{\prime}, t^{\prime}\right), \quad s, s^{\prime}, t, t^{\prime} \geq 0 .
$$

It is easy to check that, for sufficiently large $u$,

$$
2 \leq \mathbb{E}\left(\left(\zeta\left(s, t, s^{\prime}, t^{\prime}\right)\right)^{2}\right)=4-2\left(1-r_{\xi}\left(s-s^{\prime}, t-t^{\prime}\right)\right) \leq 4-\left((j-1) S_{2}\right)^{\beta} u^{-2}
$$

for any $(s, t) \in \triangle_{0}^{1} \times \triangle_{0}^{2},\left(s^{\prime}, t^{\prime}\right) \in \triangle_{0}^{1} \times \triangle_{j}^{2}$. Applying the same arguments as in the proof of Lemma 6.3 of Piterbarg (1996) we conclude that

$$
\limsup _{u \rightarrow \infty} \frac{\Sigma_{2, x}(u)}{\Psi(u) u^{2 / \beta-1}} \leq Q x\left(\mathscr{H}_{\beta}\left[0, S_{1}\right]\right)^{2} S_{2} \sum_{j \geq 1} \exp \left(-\frac{1}{8}\left(j S_{2}\right)^{\beta}\right)
$$

for some positive constant $Q$. Hence, the claim follows from (3.1)-(3.8) when $b_{3}>0$ by letting $S_{2}, S_{1} \rightarrow \infty$. When $b_{3}<0$, the same results can be obtained using similar arguments as above and the fact that

$$
1-\sigma(s, t) \geq b_{1} s^{\beta}(1+o(1))+\left(b_{2}+\frac{b_{3}}{2}\right)\left|t-t_{0}\right|^{2}(1+o(1))
$$

as $(s, t) \rightarrow\left(0, t_{0}\right)$, which is utilized for verifying (3.1). This completes the proof.

\section{Appendix A. Piterbarg's lemma for Gaussian random fields}

In order to find the asymptotics of the supremum of centered nonsmooth Gaussian processes, two crucial results are important, namely the Pickands lemma and the Piterbarg lemma. Although for experts in this field the results are well known, we would like to briefly mention them. Let $\{X(t), t \geq 0\}$ be a centered stationary Gaussian process with almost surely 
continuous sample paths and correlation function $r(t)$ which satisfies $r(t)=1-t^{\alpha}(1+o(1))$ as $t \rightarrow 0$ with $\alpha \in(0,2]$ and $r(t)<1$ for all $t>0$. In the seminal paper Pickands (1969) it was shown that, for any $T \in(0, \infty)$,

$$
\mathbb{P}\left\{\sup _{t \in[0, T]} X(t)>u\right\}=\mathscr{H}_{\alpha} T u^{2 / \alpha} \Psi(u)(1+o(1)) \quad \text { as } u \rightarrow \infty .
$$

The proof of (A.1) strongly relies on Pickands' lemma which states that

$$
\mathbb{P}\left\{\sup _{t \in\left[0, u^{-2 / \alpha} T\right]} X(t)>u\right\}=\mathscr{H}_{\alpha}[0, T] \Psi(u)(1+o(1)) \quad \text { as } u \rightarrow \infty .
$$

In the seminal contribution Piterbarg (1972), Piterbarg rigorously proved (A.1) and then extended (A.2) to a result which we refer to as the Piterbarg lemma, namely, for any constant $b>0$,

$$
\mathbb{P}\left\{\sup _{t \in\left[0, u^{-2 / \alpha} T\right]} \frac{X(t)}{1+b t^{\alpha}}>u\right\}=\mathcal{P}_{\alpha}^{b}[0, T] \Psi(u)(1+o(1)) \quad \text { as } u \rightarrow \infty .
$$

Our next result is a variant of the Piterbarg lemma for the two-dimensional case. We omit its proof since it follows exactly the same arguments as those used in the proof of Lemma 6.1 of Piterbarg (1996).

Lemma A.1. Let $\{\xi(s, t), s, t \in \mathbb{R}\}$ be a centered homogeneous Gaussian random field with covariance function

$$
r_{\xi}(s, t)=\exp \left(-|s|^{\alpha_{1}}-|t|^{\alpha_{2}}\right), \quad s, t \in \mathbb{R}, \alpha_{1}, \alpha_{2} \in(0,2] .
$$

Furthermore, let $S, T$, and $T_{0}$ be three constants such that $S, T>0$ and $T_{0} \in \mathbb{R}$. Then, for any constant $b \geq 0$ and any positive function $g(u), u \geq 0$, satisfying $\lim _{u \rightarrow \infty} g(u) / u=1$, we have

$$
\begin{gathered}
\mathbb{P}\left\{\sup _{(s, t) \in\left[0, u^{-2 / \alpha_{1}} S\right] \times\left[u^{-2 / \alpha_{2}}\right.} \frac{\xi(s, t)}{\left.T_{0}, u^{-2 / \alpha_{2}}\left(T_{0}+T\right)\right]}>g(u)\right\} \\
=\mathcal{P}_{\alpha_{1}}^{b}[0, S] \mathcal{H}_{\alpha_{2}}[0, T] \Psi(g(u))(1+o(1))
\end{gathered}
$$

as $u \rightarrow \infty$.

Remark. In the last formula we identify $\mathcal{P}_{\alpha_{1}}^{b}[0, S]$ to be $\mathscr{H}_{\alpha_{1}}[0, S]$ when $b=0$.

\section{Acknowledgements}

The authors are thankful to the anonymous referee for his/her suggestions and comments. They also kindly acknowledge partial support from the Swiss National Science Foundation Project 200021-1401633/1, and the project RARE -318984 (a Marie Curie IRSES Fellowship within the 7th European Community Framework Programme).

\section{References}

Adler, R. J. ANd TAYlor, J. E. (2007). Random Fields and Geometry. Springer, New York. Albin, J. M. P. (1990). On extremal theory for stationary processes. Ann. Prob. 18, 92-128. 
Asmussen, S. (1987). Applied Probability and Queues. John Wiley, Chichester.

Asmussen, S. And Albrecher, H. (2010). Ruin Probabilities, 2nd edn. World Scientific, Hackensack, NJ.

Awad, H. And Glynn, P. (2009). Conditional limit theorems for regulated fractional Brownian motion. Ann. Appl. Prob. 19, 2102-2136.

DȩBICKI, K. (2002). Ruin probability for Gaussian integrated processes. Stoch. Process. Appl. 98, 151-174.

DȩBiCKI, K. AND MAndJES, M. (2003). Exact overflow asymptotics for queues with many Gaussian inputs. J. Appl. Prob. 40, 704-720.

DȩBicki, K. And MandJes, M. (2011). Open problems in Gaussian fluid queueing theory. Queueing Systems 68, 267-273.

Dȩbicki, K. and Tabiś, K. (2011). Extremes of the time-average stationary Gaussian processes. Stoch. Process. Appl. 121, 2049-2063.

DȩBicki, K., Hashorva, E. AND Ji, L. (2014). Gaussian risk models with financial constraints. Scand. Actuarial J. DOI: $10.1080 / 03461238.2013 .850442$.

DȩBicki, K., Michna, Z. And Rolski, T. (2003). Simulation of the asymptotic constant in some fluid models. Stoch. Models 19, 407-423.

DieKer, A. B. AND YAKIR, B. (2014). On asymptotic constants in the theory of extremes for Gaussian processes. Bernoulli 20, 1600-1619.

Duncan, T. E. And Jin, Y. (2008). Maximum queue length of a fluid model with an aggregated fractional Brownian input. In Markov Processes and Related Topics: a Festschrift for Thomas G. Kurtz (Inst. Math. Statist. Collect. 4), Institute of Mathematical Statistics, Beachwood, OH, pp. 235-251.

Embrechts, P., Klüpelberg, C. And Mikosch, T. (1997). Modelling Extremal Events. For Insurance and Finance. Springer, Berlin.

Griffin, P. S. (2013). Convolution equivalent Lévy processes and first passage times. Ann. Appl. Prob. 23, $1506-1543$.

Griffin, P. S. AND Maller, R. A. (2012). Path decomposition of ruinous behavior for a general Lévy insurance risk process. Ann. Appl. Prob. 22, 1411-1449.

Griffin, P. S., Maller, R. A. And Roberts, D. (2013). Finite time ruin probabilities for tempered stable insurance risk processes. Insurance Math. Econom. 53, 478-489.

Harrison, J. M. (1985). Brownian Motion and Stochastic Flow Systems. John Wiley, New York.

Hashorva, E., Ji, L. AND Piterbarg, V. I. (2013). On the supremum of $\gamma$-reflected processes with fractional Brownian motion as input. Stoch. Process. Appl. 123, 4111-4127.

Hüsler, J. And Piterbarg, V. (1999). Extremes of a certain class of Gaussian processes. Stoch. Process. Appl. 83, $257-271$.

Hüsler, J. And Piterbarg, V. (2008). A limit theorem for the time of ruin in a Gaussian ruin problem. Stoch. Process. Appl. 118, 2014-2021.

HÜSLER, J. ANd Zhang, Y. (2008). On first and last ruin times of Gaussian processes. Statist. Prob. Lett. 78, 1230-1235.

Kozachenko, Y., Melnikov, A. And Mishura, Y. (2014). On drift parameter estimation in models with fractional Brownian motion. Statistics DOI: 10.1080/02331888.2014.907294.

MandJes, M. (2007). Large Deviations for Gaussian Queues. John Wiley, Chichester.

PICKANDS, J., III (1969). Asymptotic properties of the maximum in a stationary Gaussian process. Trans. Amer. Math. Soc. 145, 75-86.

Piterbarg, V. I. (1972). On the paper by J. Pickands 'Upcrosssing probabilities for stationary Gaussian processes'. Vestnik Moscov. Univ. Ser. I Mat. Meh. 27, 25-30 (in Russian). English translation: Moscow Univ. Math. Bull. 27, 19-23.

Piterbarg, V. I. (1996). Asymptotic Methods in the Theory of Gaussian Processes and Fields (Transl. Math. Monogr. 148) American Mathematical Society, Providence, RI.

Piterbarg, V. I. (2001). Large deviations of a storage process with fractional Browanian motion as input. Extremes 4, 147-164.

Whitт, W. (2002). Stochastic-Process Limits. An Introduction to Stochastic-Process Limits and Their Application to Queues. Springer, New York.

ZeEvi, A. J. AND GlynN, P. W. (2000). On the maximum workload of a queue fed by fractional Brownian motion. Ann. Appl. Prob. 10, 1084-1099. 